\title{
Adaptive and Optimization of Personalized Information Retrieval Model in Semantic Web
}

\author{
J.I.Christy Eunaicy, S.Suguna
}

\begin{abstract}
The recognition of user's visited set of web pages for the prediction of web page is a key drawback. Thus the work is employed with the web access log files which is stored in the server. To understand the user interest patterns, the web access log files are extracted that depicts the user behavior. Various applications can be employed to predicting user's behavior while serving the web. During this work, the proposed framework analyze the user usage, reinforced the content and the content retrieved with the semantic manner. The semantic information retrieval supported the user access pages are preprocessed and the web log data of the particular user is analyzed to identify the user profile. Then the retrieved information is graded with clustering the semantic content based results. The ranked content is then analyzed with the user profile to produce an optimized search results for the users based on the user classification.
\end{abstract}

Index Terms: Semantic Web, Pattern Extraction, Web Content Mining, Web Usage Mining, Semantic Cluster

\section{INTRODUCTION}

In the present situation volatile development of knowledge realistic on web makes the clients to get to the data step by step. It turns out to be somewhat increasingly hard for clients to get to significant information with proficiency. Investigating and demonstrating navigation of web performance is valuable in understanding requests for online clients. Web mining is an information extraction system to analyze and obtain valuable information from web data. Web mining might be characterized in to three unique classes: web content mining, web usage mining and web structure mining.

Web content mining is that the revelation of content from web documents like content, image, video, audio and so on. Web structure mining focus on investigating the physical associated structure of sites. Web usage mining evaluates the perusing action. The web usage data comprises of information from weblog. The accessing information of the users from the websites is stored in $\log$. The log contains organization of user's exchanges that are in many cases refreshed at whatever point the client gets to the site. The expectation of user behavior will be known exclusively through logs. The weblog contains unstructured configuration, thusly convert to raw weblog to prepared weblog utilizing data preprocessing, the data preprocessing incorporates learning cleaning, User

Revised Manuscript Received on 16 September 22, 2019.

J.I. Christy Eunaicy, Research Scholar, Bharathiar University, Coimbatore, India.

Dr.S.Suguna, Asst.Professor, Dept of Computer Science. Sri Meenakshi Govt college for Women, Madurai, India
Identification, Session Identification, content recovery and path completion to instigate client pattern of navigation.

Essentially the web usage mining categorizes the procedure into three classes. The essential is preprocessing state during which user sessions are deduced from log data. The second stage of this process is searching patterns in the information of the standard processing of data techniques, such as sequent pattern mining or association rule mining. The third stage of this process is an information extracts from the domain knowledge and the structures of the website is employed to search and mining the patterns. Connections among pages and the content similarity of pages offer proof that pages are associated.

From the disordered human acceptable web information semantic web is the way to successfully and proficiently making a machine-reasonable, questioned, data and learning layer. On the off chance that computer can comprehend the importance behind the data, it can realize what the system are intense on and it help us better to discover what the system need. Since the semantic web by and large spotlights on the information. Information in the Semantic Web is all around characterized and connected in a manner that can be utilized for progressively interesting revelation, robotization. The idea of most information on the Web is unstructured that just comprehend by people, the measure of information is exceptionally enormous on the web that handled proficiently by machines. The objective of the Semantic Web is to create permitting benchmarks and advances intended for both user and machines reasonable.

\section{RELATED REVIEW}

Web usage mining anticipate the behavior of the user navigation dependent on the inclinations in site, User access path methodology utilizes data preprocessing Patel and Renata Ivancsy [1] recommended that the weblog contains basic log design, so convert to expected weblog to prepared weblog utilizing data preprocessing, the data preprocessing method contains Data Cleaning, User Identification, Session Identification, retrieval of information and way finishing to get user navigation design. In the wake of getting the processed $\log$, the given $\log$ is changed over in to pattern sequences dependent on client's pattern.

Hong Cheng and Xifeng Yan [2] indicated user get the prepared log from simple $\log$, at that point web traversal pattern is dissect to arrangement calculation to characterize the preprocessed log into most extreme forward sequence. The techniques characterize the Frequent Sequence, Semi-visit Sequence and Infrequent Sequence. 
Kerana Hanirex and Dorai Rangaswamy [3] indicated characterization of continuous item sets, semi-visit item sets and In-visit item sets and database exchanges into groups. Clusters are assembled dependent on the comparable traversal pattern. At that point it finds the successive item sets, semi-visit item sets and In-visit item sets, the clusters productivity is improved.

Joachims, Juhne [4] and Pazzani and Billsus [5] demonstrated the instances of users recommend connections of every client. The visitor visit the web server dependent on user enthusiasm, by whenever the program will demonstrate the user propose site dependent on client interest. The browser will pursue the successive visited items, at whatever point the client will visit some different pages that will demonstrate much of the time of webserver connections, and afterward no one but user can pursue the consistently visited connections.

Jagan and Rajagopalan [6] depict the web usage extraction and illustrations utilized for giving personalization on the web. In this paper centered the information preprocessing and pattern investigation on the web and utilizing the association

Anand [7] depicts a web use subtleties and gives them the devices to comprehend the online behavior of their high school kids. Singh A.P. [8] Different sorts of web usage mining techniques with their fundamental models and ideas are given. Notwithstanding that, for finding the hidden patterns from web access log documents another model dependent on visual clustering is additionally recommended. Mishra and Choubey [9] portray the FP growth algorithm is acquiring a most every now and again get to ways and pages from the web log information and giving significant data to user behavior. Zubi and Riani [10] examines the utilization of web mining methods is utilized to order the site page's category as indicated by user visits. This order is comprehends the web user behavior. The classification and association rule strategies for finding the interesting data from browsing patterns. Avneet Saluja et al. [11] in their work, user future request prediction utilizing web log records and client data is given. The motivation behind the application is to give a benchmark to assessing a different techniques utilized before, a present and which can be utilized in a future to limit the search time of a user on the system.

\section{MOTIVATION AND PROBLEM STATEMENT}

In the recent occasions an ever increasing number of user are utilizing the internet providers for their need. This aid propose an exploration in mining valuable data and client's enthusiasm from the web utilizing mining methods. To mining the user's advantage assessment the best helpful property is the Web Logs of the users. Since web logs are refreshed each time the user visits a specific site, so it is considered as moving information. In this manner the work is motivated to advancement of the usage based content mining and suggestion with the work of Semantic Web. The primary inspiration for our work is given as pursues:

- The challenge of the semantic web mining in the suggestion framework to give learning of personalization of the users is engaged. decreases the quantity of exchange in the database and the rule mining techniques.

- To build up a conventional system that coordinates semantic based information retrieval to usage extraction for the proficient mining technique and suggestion.

- A structure for the personalized proposal framework dependent on consolidated user usage profile and field knowledge that incorporates development of suggestions.

- The challenge of the issues in providing personalized semantic based web get to activities and consequently find the associations among the activities to assume personalized user usage learning with the categorization.

\section{PROPOSED METHODOLOGY}

The proposed framework means to search out the web client conduct from clients through the web server log documents. Inside the model, the user demands the website page and interconnections naturally get keep in a server log record. All mentioned site connections get put away and kept up by the user. A noisier and messy information's present inside the log records. Preprocessing steps are important to organize of the commotion, absent and excess data. During preprocessing, the user recovers persistent web connection used by users, the hypertext transfer protocol demand pages, user recognizable in a few users and sessions is known and path fulfillment. These strategies acclimated predict the behavior of the users.

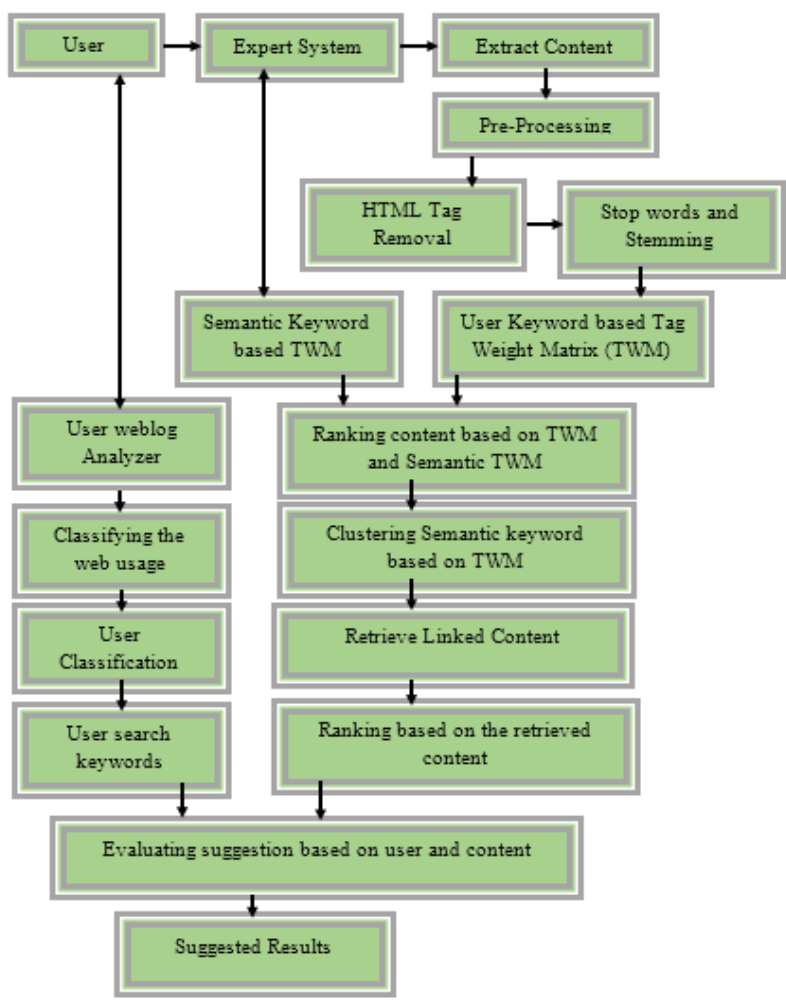

Fig. 1. Proposed Model Architecture

In the above Fig. 1, the overall framework Adaptive Framework for Personalized and Optimized Information Retrieval (AFPOIR) for the user based recommendation system is illustrated. The main objective of this framework is to provide recommendation for the users based on their behavior and semantic based suggestions. 
The aim of the semantic based suggestion system would provide more relevant recommendation based on the user's category using user classification. This framework includes the following levels for the recommendation evaluation.

\section{Expert System}

The proposed framework pretentions of the visiting of user's web interface and whole crawled information will be will at that point be handled to evacuate insignificant information. Various systems are then employed on cleaned $\log$ record to give successful personalized suggestion. In this stage the whole slithered information of the considerable number of clients, which comprises of all the site pages visited is gathered and maintained in a log file. This work utilizes common log document configuration to record the information. Following significant properties in particular IP address, time, date, status code, URL, method (GET and POST), user agent and Referrer URL are considered for investigation.

\subsection{Content Retrieval and Preprocessing}

The content of the various sources of web pages are gathered. It is commonly utilized as base of web mining system. The preprocessing stage in Web Usage Mining procedure includes cleaning and organizing information and organizes up for the pattern revelation stage. It is of various types: structure pre-preparing, content pre-processing and usage pre-processing. Data extraction undercover the gathering of document into a data that is more clear and access. The principle point of data extraction is to choose important actuality from document as not at all like in the information retrieval used to choose relevant document.

Data extraction interprets the structure of illustration of record while information retrieval sees just content in document. Data extraction can use to alter the ordering procedure which is really a work of information retrieval process.

The pre-processing of data part was performed utilizing a condensed log file, which was "cleaned" by evacuating all inadequate, asymmetrical, and missing data from the unique common log record. After the underlying preprocessing, a session filter was employed to the decreased $\log$ record for feature extractions. The point of the filter was to entire all user demands inside a session into a private collection of factors.

Extraction includes all the retrieval of information programs that are not intended to safeguard the source page [13]. The accompanying advances are performed for the content extraction:

- A crawler begins off by retaining a preliminary collection of URLs, in a column, any place all URLs to be recovered are kept and organized.

- Transformation grasps all preparing where the input and the output are HTML pages.

- DOM (Document Object Model) analyzer characterizes the indication of blocks in website pages. The appropriate pages given out by the web crawler are characterized to in a type of DOM tree HTML DOM is in a tree structure, ordinarily called a HTML DOM tree [13].

- Then the Stop Words Elimination intends to make the content look heavier and less significant for gathered and sustained in a log document. The log document

investigation by expelling stop words to decrease the dimensionality of term space [13].

\subsection{Tag Weighted Matrix (TWM)}

In the next level of this system, is to construct a matrix known to be Tag Weighted Matrix (TWM). The TWM is used to obtain the tag-wise content to evaluate the weighting. The TWM contains the words or content includes in the HTML tags for the evaluation of weighting based on the user search keywords. It is dynamically constructed matrix to identify the tag contents and the linked content of the user behavior. In this specific situation, the TWM Matrix is utilized describe the related keywords from the content of website pages. The massive measure of web reports has brought about tribulations for data retrieval essential to the truth that the search results are of less consequence to the user [12]. In this work, the framework propose a unique thought for finding near duplicates of an input website page, from an immense warehouse.

\section{Table I. Tag Weighted Matrix}

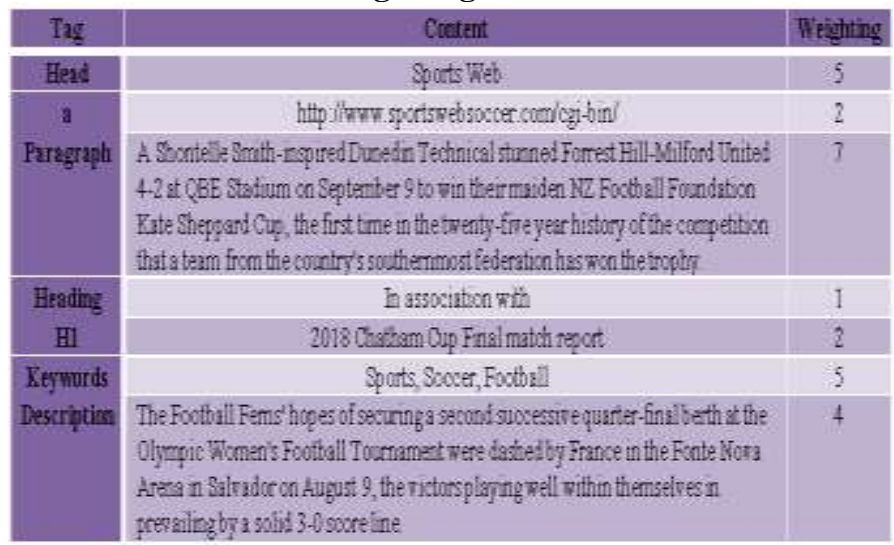

The above Table I depicts that a sample representation of TWM for a user search keyword. The table illustrates that tags are separated and extract the contents included in the particular tag of the obtained search results for the user search keyword. The weighting for the particular tag and content is evaluated by using the search keyword occurrence. Sample weighting evaluation for TWM based on user search keyword shown in Table II.

Table II. Sample Weighting TWM

\begin{tabular}{|c|c|c|c|}
\hline Keywords/Results & r1 & r2 & r3 \\
\hline \multirow[t]{2}{*}{$\mathrm{k} 1$} & 0 & $W k 1+2$ & Wk1r2 \\
\hline & & $\overline{\text { SWkir2 }}$ & $\overline{\sum W k i r 2}$ \\
\hline \multirow[t]{2}{*}{$\mathrm{k} 2$} & $W k 2+1$ & $W k 2+2$ & $W k 2+3$ \\
\hline & $\overline{\Sigma W \text { kir1 }}$ & $\overline{\sum W k i r 2}$ & $\overline{\sum W k i r s}$ \\
\hline $\mathrm{k} 3$ & 0 & $\frac{W k 3+2}{\sum W k i r 2}$ & 0 \\
\hline $\mathrm{k} 4$ & $\frac{W k 4 r^{-1}}{F W_{k i r 1}}$ & 0 & $\frac{W k 4+3}{\sum W k i r 3}$ \\
\hline
\end{tabular}

The table depicts that the sample weighting matrix based on the tag contents is denoted as keywords $\mathrm{k}=\{\mathrm{k} 1, \mathrm{k} 2, \mathrm{k} 3, \ldots \ldots\}$ and number of search results as $\mathrm{r}=\{\mathrm{r} 1, \mathrm{r} 2, \mathrm{r} 3, \ldots \ldots\}$. Then the weighting $\mathrm{W}$ for the keyword with the results is represented with respect to the search keyword and search results. The evaluation is carried out for the collection of URL list from the results and construct TWM matrix for content extracted. 


\subsection{Semantic based TWM}

After computing the weighted TWM, the obtained matrix if further constructed to make semantic based TWM (S_TWM). The semantic keywords are buildup with the various users' search keywords. S_TWM emphasis around storing semantic features of a record, for example, creator name, document content, association concerned or area of work. This data is recorded in semantic data table and is well-mined to get accommodating information/pattern, for example, extra expansion of documents in a particular situation indicates more research/advancement affinity of users here. Additionally, least gone to zone can likewise be found.

The sample S_TWM is represented in Table III:

Table III. Semantic based Tag Weighted Matrix for the

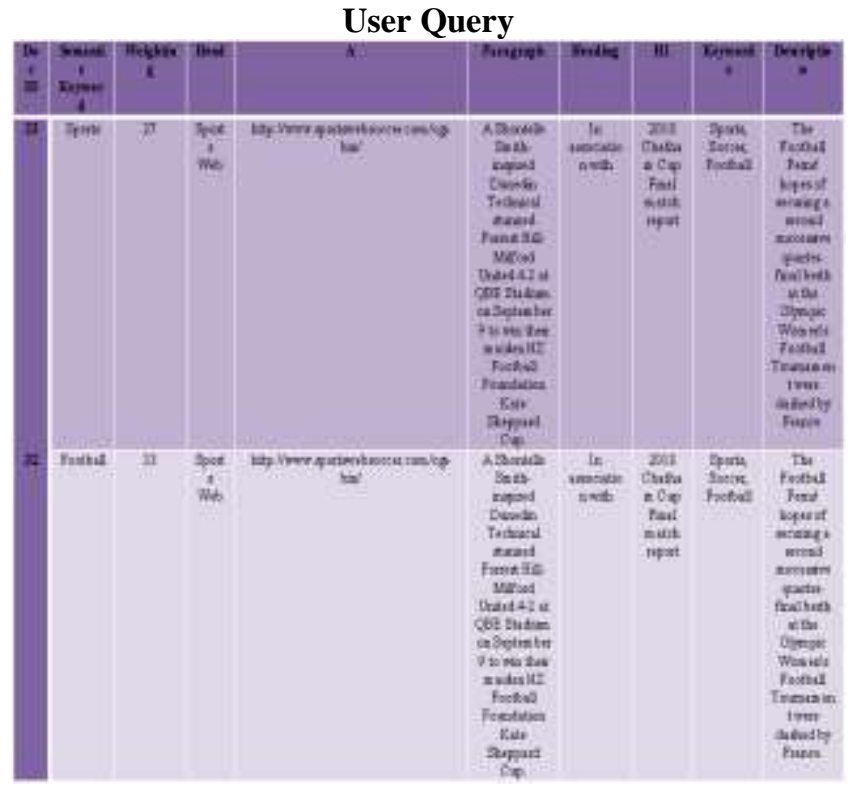

\subsection{Ranking and Clustering based on Semantic Content}

Cluster based Ranking approach directly generates cluster with ranking. Ranking conveyance of tags in every cluster ought to be very not the same as one another. At first the records are grouped into clusters. At that point the tags are ranked inside each group. From that point forward, an integrated model is utilized to disapprove each tag into a $\mathrm{K}$-dimensional vector, where each element is a section stable with respect to a cluster. Each element is estimated by rank dissemination. Tags at that point are reassigned to the closest cluster under the new keywords from the users. Thus, the nature of tag clustering is improved. Moreover, tag ranking outcomes would thus be able to be improved further by these intense tag clustering. Taking all things together, as opposed to integrating ranking and clustering in a two phase technique simply like the top of the line, segregation, the work propose a methodology which can commonly upgrade the nature of clustering and ranking known to be Semantic Content Clustering based Ranking Approach (SCC_RA).

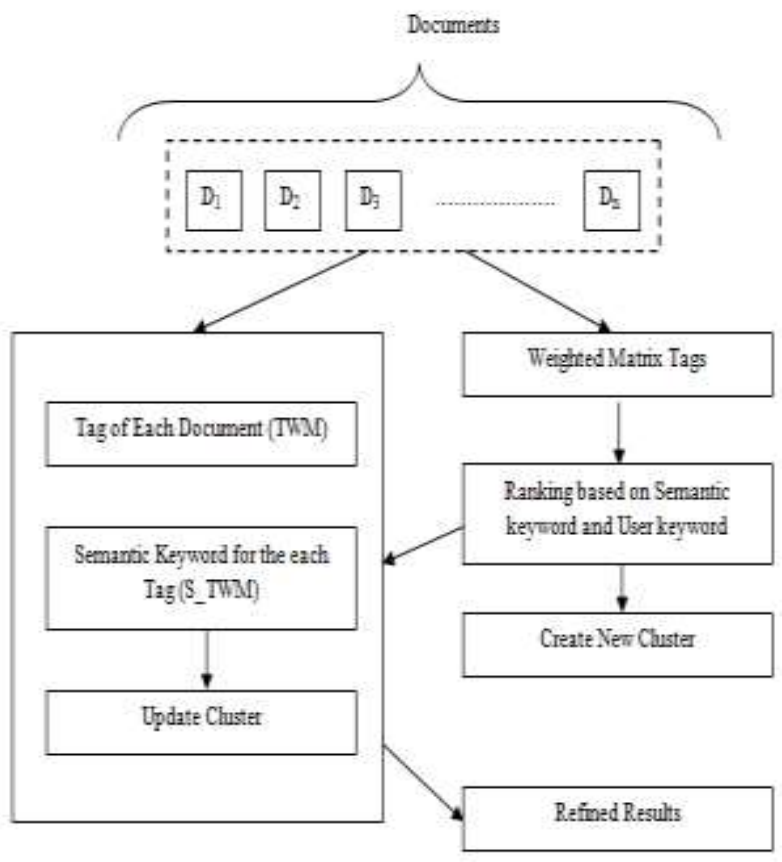

Fig. 2. Ranking and Clustering of Semantic Content

That is, tag ranking can improve the exhibition of clustering the tag and the acquired product of tag clustering can further upgrade the presentation of ranking the tag. The inspiration of the methodology is that, for each tag cluster, which structures a theme, the keyword rank conditional on this point theme ought to be exceptionally distinct, and very not quite the same as the keyword rank in other point theme. Thus, employing either ranking or clustering over the whole record set regularly brings about deficient, or at times rather one-sided, analytical outcomes. For example, tags ranking over the full file set while not thinking about which clusters they have a place with typically brings about irrelevant outcomes.

Table IV. Ranking based S_TWM

\begin{tabular}{|l|l|l|l|l|l|}
\hline ID & $\begin{array}{l}\text { Semantic } \\
\text { Keyword }\end{array}$ & $\begin{array}{l}\text { User } \\
\text { Keyword }\end{array}$ & $\begin{array}{l}\text { Doc } \\
\text { ID }\end{array}$ & Weighting & Ranking \\
\hline $\mathbf{1}$ & Sports & Sports event & 23 & 27 & 11 \\
\hline $\mathbf{2}$ & Football & $\begin{array}{l}\text { Outdoor } \\
\text { sports }\end{array}$ & 32 & 33 & 12 \\
\hline $\mathbf{3}$ & Indoor Sports & Table tennis & 12 & 23 & 15 \\
\hline
\end{tabular}

\section{Algorithm SCC_RA}

\{

Input: S_TWM - Semantic based Tag Weighted Martix

Output: $\mathrm{C}_{\text {Semantic }}-$ Semantic based Clusters

Obtain the initial for cluster $\mathrm{C}=1,2 \ldots . \mathrm{k}$

Get the number of documents $D=d_{1}, d 2, \ldots, d_{n}$

For $\mathrm{i}=1$ to $\mathrm{n}$ in document $\mathrm{D}$

\{

Read the Tag from document $d_{i}$

Obtain TWM Matrix for each tag of the document Compute weight $\mathrm{W}_{\mathrm{i}}=\frac{W_{\mathrm{t} 1+2}}{\sum W_{\mathrm{nir} 2}}$ for each tag in the Matrix

Obtain semantic based TWM for document $\mathrm{d}_{\mathrm{i}}$

Compute ranking $\mathrm{R}$ based on the semantic content occurrence 
$\mathrm{R}=\sum_{n}^{i=1} \frac{\text { Keywora }_{\text {new }}}{\text { Keywora }_{n}-\text { keywora }_{\mathrm{i}}}$

Construct Ranking based S_TWM matrix

For each Tag $\mathrm{t}$ in $\mathrm{T}$

\{

For each $\mathrm{k}=1$ to $\mathrm{K} / /$ Cluster formation

\{

Compute similarity value $\operatorname{Sim}_{\tau}=\left(t_{k}, C_{k}\right)$

\}

Assign to the cluster $\mathrm{C}_{\mathrm{k}}$

\}

\}

\}

\subsection{User Web Log Analyzer}

The user behavior information are obtained from the Web Log Files. The file includes the addressed requests to the web servers. These records are stored with the record ordering and various web servers sustain different types of data.

The web log analysis is performed by Web log analyzer (WLA). WLA comprise of log cleanse, session discovery term spread and page consolidating methods utilized for web log examination. Each log entry includes IP Address, password, name, URL, request method, access time, referrer, transmission bytes, status code and protocol. Since each log section isn't useful, web logs are cleansed by sifting through all images, video, and sound requests and failed page requests. A session of user is characterized as a group of clicks over a limited time by a user. Each user is thought to be unambiguously arranged out by the IP address recorded in the request of protocol. The entire web server log will be outlined as a group of sessions. Access URL is the essential site page visited in a session and track referrer is the referrer of the access URL. The information present in the log document can't be utilized for what it's worth for the mining strategy. So the content of the log record should be cleaned during this preprocessing step. The undesirable information are evacuated and a decreased log document is acquired [12].

\section{User Identification}

In data cleaning, the user can be categorized from the records. The identification of user from the obtained log information like server name, IP, user agent, etc, are evaluated. The phase of data preprocessing that fetches the page sequences based on user's activities or script accesses.

The users are classified with the selection criteria using classification technique [12]. The user's profile is created with analysis of sessions maintained with the one user by another. The users visited path, IP address and time stamp is obtained to construct a user's profile is shown in Table V. The user profile is constructed dynamically with the user's search requests. Then the dynamically constructed user profile is then trained to the classifier to classify the user to provide optimized results for their searching.
Table V. Sample User Identification

\begin{tabular}{|c|c|c|}
\hline $\begin{array}{l}0 s= \\
1\end{array}$ & 2142113422 & 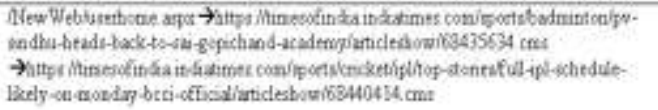 \\
\hline$\frac{\text { User }}{2}$ & ${ }_{0}^{49} 20511311$ & 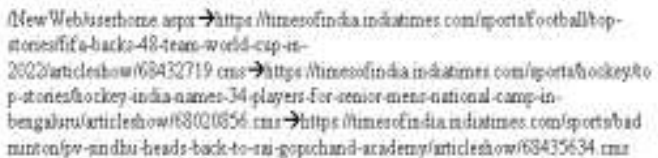 \\
\hline $\begin{array}{l}\text { Deer } \\
3\end{array}$ & 1039620170 & 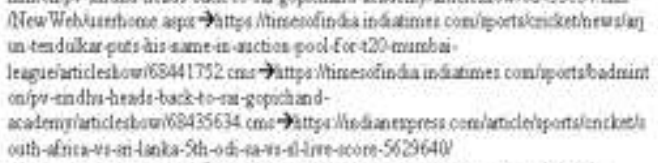 \\
\hline $\begin{array}{l}\text { Vaer } \\
4\end{array}$ & $\begin{array}{l}185.136 .156 .1 \\
55\end{array}$ & 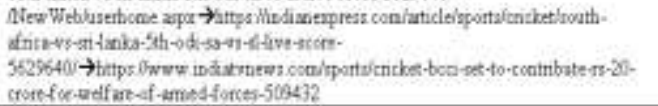 \\
\hline
\end{tabular}

\section{Session Identification}

In this section, the identification of the user's navigation pattern acquisition based on session is performed after the cleaning and identification of the user. The following are the process of pattern navigation is clustered the session.

In the proposed method a vector is constructed from which sessions are identified. User's traversal is the input to the method. Vector consists of the web pages and users and their sessions identified qq is shown in Table VI. Browsing time for a particular page is determined by finding the differences between the time fields of two consecutive entries of a same user [12].

Table VI. Sample Session Identification Vector

\begin{tabular}{llccccc}
\hline Users & Sessions & \multicolumn{5}{c}{ URLs } \\
\hline & & URL1 & URL2 & URL3 & URL4 & URL5 \\
User1 & S1 & 1 & 0 & 3 & 10 & 0 \\
User1 & S2 & 1 & 2 & 0 & 0 & 0 \\
User1 & S3 & 0 & 0 & 10 & 50 & 0 \\
User2 & S1 & 0 & 20 & 0 & 0 & 0 \\
User2 & S2 & 1 & 10 & 0 & 2 & 0 \\
User2 & S3 & 1 & 0 & 1 & 1 & 1 \\
User3 & S1 & 0 & 11 & 0 & 12 & 0 \\
User3 & S2 & 1 & 0 & 10 & 10 & 0 \\
User3 & S3 & 1 & 10 & 10 & 0 & 1 \\
User4 & S1 & 0 & 0 & 0 & 0 & 0 \\
User4 & S2 & 1 & 10 & 0 & 0 & 1 \\
User4 & S3 & 1 & 2 & 1 & 0 & 0 \\
\hline
\end{tabular}

\subsection{User Behavior Classification and Recommendation} Model

The intention is to improve the ease of use of the web information and to utilize the systems on the web applications like, prefetching and storing, personalization and so forth. For evaluation the executives, the results of web utilization extraction are regularly used for target commercial, upgrading website configuration, improving fulfillment of client, directing the technique optimal of the scheme, and promoting inspection and so forth. Anticipating the user's pattern of browsing is one of web utilization mining method. For this reason, it is expected to recognize the clients' browsing pattern by implies that of examining the web information or web log documents. Expecting the accurate user's next requirements is as indicated by the previous associated actions. 
There are numerous benefits to utilize the expectation, for instance, personalization, building right site, improving advertising methodology, getting promoting information, determining business sector ideas, and expanding the focused quality of events and so forth.

In this the proposed TCOUB classification for the optimization of the user's search results based on user behavior is described. When identifying user sessions, the various techniques of web usage pattern discovery are applied in order to observe interesting and helpful patterns. There are many forms of access pattern mining which will be performed depending on the needs of the analyst. In order to attain this optimized user classification is used. TCOUB algorithm integrates the Tri-Class Classification with the optimization [12].

In this section of the work, the user behavior is obtained with the help of user web log analyzer to categorize the user behavior based on their usage. The most aspect of this method is to create an efficient recommendation system for the multi-users in the web. The user's search might vary from one user to another user based on their keywords for searching. So in this section, the extracted user log information are further processed for user classification shown in Table VII.

Table VII. User Classification based on Behavior

\begin{tabular}{|c|l|c|}
\hline User Class & $\begin{array}{l}\text { User Description } \\
\text { Frequent Users }\end{array}$ & $\begin{array}{l}\text { User Classification } \\
\text { ID }\end{array}$ \\
$\begin{array}{l}\text { User searches } \\
\text { more frequently } \\
\text { and access to the } \\
\text { particular web } \\
\text { usage }\end{array}$ & 1 \\
\hline Exceptional Users & $\begin{array}{l}\text { User who searches } \\
\text { and access to the } \\
\text { particular usage } \\
\text { rarely }\end{array}$ & 0 \\
\hline Indeterminate & $\begin{array}{l}\text { User who doesn't } \\
\text { searches and } \\
\text { access to the } \\
\text { particular usage }\end{array}$ \\
\hline
\end{tabular}

\subsection{Evaluation of Recommendation System}

After the classification of the users, the results for the search keywords are provided with the user class. The results that are recommended for the indeterminate and exceptional users which are suggested based on the frequent users searching profile. The frequent users are suggested with their search profile and the suggestions are made for the other users based on their profile and frequent user profile. Thus the system provides optimized results based on the users profile.

\section{PERFORMANCE EVALUATION \& RESULTS}

In this section, the performance evaluation for the semantic based content mining recommendation for the web usage. The time consumption of the clustering techniques is shown in Fig. 3.

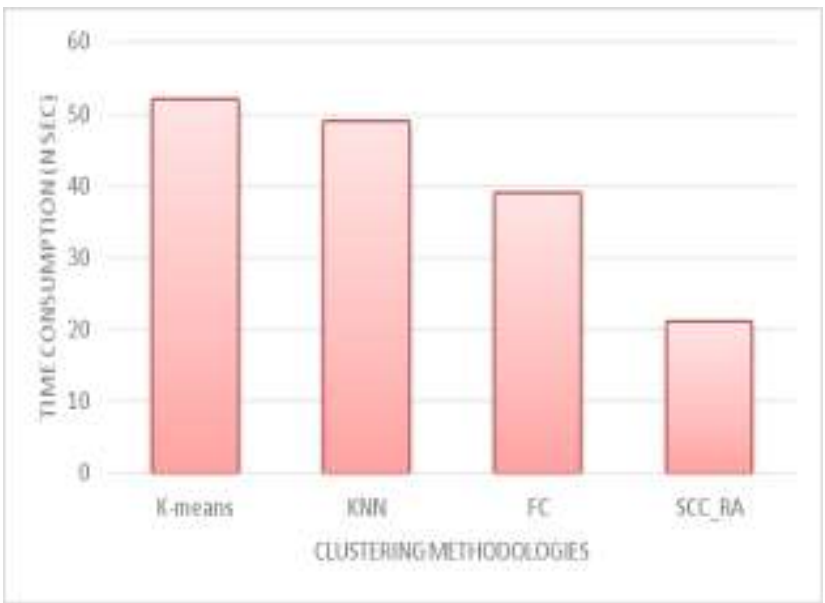

Fig. 3. Time Consumption of the Clustering

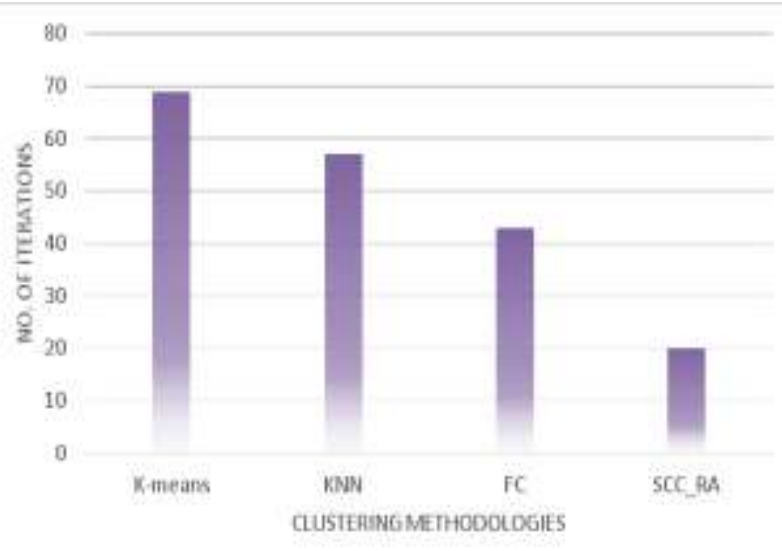

Fig. 4. Number of Iterations for the Clustering Methodologies

The number of iterations for the clustering methodologies is shown in Fig. 4. The Figure depicts that proposed SCC_RA less number of iterations than other methodologies.

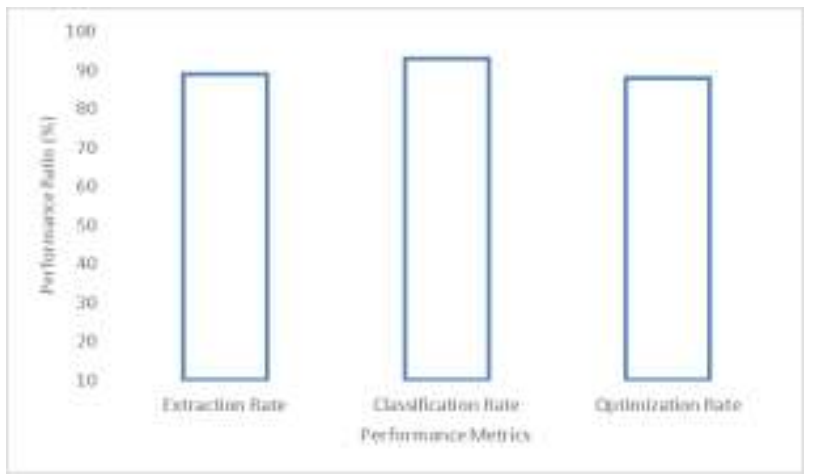

Fig. 5. Performance Metrics Evaluation

The performance metrics evaluation such as extraction rate, classification rate and optimization rate for the proposed methodology is illustrated in Fig. 5. The figure depicts that rate of the performance evaluation provides reasonably high for all the metrics while prediction. 


\section{CONCLUSION}

The key intention of the work is to propose a novel framework for the recommendation system based on the user behavior with semantic based web technique. The semantic based information retrieval system that integrates the concept of web content mining and web usage mining for the personalized recommendation system. The work focuses on the web usage mining based personalized recommendation with semantic web to overcome the challenges of content and usage mining. This recommendation system is employed to propose a generic framework for the efficient information retrieval. The framework that constructs a user profile with the user access log information that integrates with the semantic content mining for the recommendation. The user profile that classifies the user based on their access criteria. Then the content mining is employed with the semantic based content organization with Semantic TWM. With the integration of these user profile and semantic content mining, the recommendation for the personalized user become optimized.

\section{REFERENCES}

1. Ketul B. Patel, Dr. A. R. Patel, "Process of web usage mining to find interesting patterns from web usage data, "www. ijctonline. com vol. 3, no. 1, Aug 2012.

2. Hong Cheng, Xifeng Yan, Jiawei HanIncSpan: Incremental Mining of Sequential Patterns in large database.

3. Kerana Hanirex.D, Dr. M. A. Dorai Rangaswamy International Journal on Computer Science and Engineering, "Efficient Algorithm for Mining Frequent Itemsets Using Clustering Technique, "vol. 3, no. 3, March 2011.

4. Joachims.T, D. Freitag, and T. Mitchell. WebWatcher: A tour guide for the World Wide Web. In Proc. 15th Intl Joint Conf. on Art. Int., 1997.

5. Pazzani.M.J. and D. Billsus. Adaptive web site agentsIn Proc. 3rd Intl. Conf. on Autonomous Agents, 1999.

6. Jagan.S, and S.P. Rajagopalan, "A survey on web personalization of web usage mining", IRJET International Research Journal of Engineering and Technology, 2015.

7. Anand.N, "Effective prediction of kid's behavior based on internet use", International Journal of Information and Computation Technology, 2014.

8. Singh.A.P., R. C. Jain, "A Survey on Different Phases of Web Usage Mining for Anomaly User Behavior Investigation", IJETTCS - International Journal of Emerging Trends \& Technology in Computer Science, Vol 3, 2014.

9. Mishra.R, A. Choubey, "Discovery of Frequent Patterns from Web Log Data by using FP-Growth algorithm for Web Usage Mining", International Journal of Advanced Research in Computer Science and Software Engineering, Vol 2, 2012

10. Zubi.Z.S., M.S. Riani, “Applying web mining application for user behavior understanding", Recent Advances in Image, Audio and Signal Processing

11. Saluja, B. Gour, and L. Singh., "Web Usage Mining Approaches for User's Request Prediction: A Survey" IJCSIT-International Journal of Computer Science and Information Technologies, Vol. 6 (3), 2015

12. J.I. Christy Eunaicy, S. Suguna, "User Classification and Optimized Recommendation System based on User Profile Generation Using Web Usage Mining”, Journal of Advanced Research in Dynamical \& Control Systems, Vol. 11, 06-Special Issue, 2019

13. J.I. Christy Eunaicy, S. Suguna , "Personalization of the User Behavior Patterns based on the Web Content Mining " , International Journal of Research ISSN NO:2236-6124, Volume VIII, Issue VI, June 2019

\section{AUTHORS PROFILE}

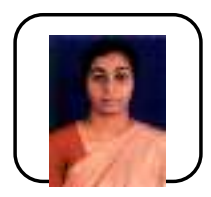

Mrs. J.I. Christy Euaicy is currently a Ph.D Research scholar from Bharathiar University, TamilNadu, India. . She was awarded with Master of Computer Applications and M.Phil from Madurai Kamaraj University, TamilNadu, India. Her research area focuses on Web Mining and Semantic web. She had been worked as Asst Professor in various reputed Institutions in Abroad and India. She has published many research journals in web mining

Dr.S. Suguna is an Assistant Professor in Computer Science, Sri Meenakshi Govt college for women, Madurai, Tamilnadu, India. Her area of

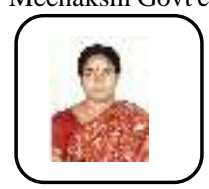
research interests include Web Mining, Semantic Web and Cloud Computing. She obtained her Ph.D from Mother Teresa University, Kodaikanal, Tamilnadu. She published many research papers which contributed significantly to the development of her field. She is also a research supervisor recognized by reputed universities. 